\title{
Untersuchungen zur kleinräumigen Bevölkerungs-, Wohn- und Arbeitsplatzstruktur der Stadt Bern
}

\section{Einleitung}

In der Stadtplanung und verschiedenen anderen Verwaltungszweigen einer Stadt läßt sich in den letzten Jahren und Jahrzehnten eine ständig größere Nachfrage nach statistischen Grundlagen feststellen. Die Ausdehnung erfolgte dabei nicht nur in Richtung einer weiteren sachlichen Differenzierung der Daten, sondern vor allem auch in Richtung einer weitergehenden räumlichen Unterteilung der Stadt. Viele Probleme können heute nicht mehr aus dem Blickwinkel der Stadt als ganzes angegangen werden, sondern verlangen nach einer räumlichen Differenzierung in mehr oder weniger kleine Einheiten. Das Statistische Amt der Stadt Bern bemüht sich deshalb seit einigen Jahren, vermehrtes Gewicht auf statistische Mikroanalysen zu legen.

Wegen des großen Auszählungsaufwandes sind diese aber erst seit der Anwendung der elektronischen Datenverarbeitung in der Statistik in größerem Umfange möglich. Im weiteren eignet sich vor allem das Datenmaterial der gesamtschweizerischen Großzählungen dafür, da selten sonst soviele Merkmale über die Bevölkerung, die Wohnungen oder die Arbeitsstätten in einer Datei vorhanden sind. Tab. 1 gibt einen Überblick über die heute vorhandenen mikrostatistischen Daten für Stadt und Region Bern.

\section{Die Gliederung der Stadt Bern}

Die Stadt Bern wird für statistische Zwecke seit etwa 1920 in sechs Stadtteile und 30 (seit 1962 32) Statistische Bezirke aufgegliedert. Diese Einteilung ist historisch zu erklären und genügt heute für viele Zwecke nicht mehr. So geht die Abgrenzung der fünf Bezirke der Innenstadt auf die Stadtentwicklungsphasen im Mittelalter und die Benennung auf die Zeit der französischen Besetzung (8. April 1798) zurück. Die Bezirke 29-32 bildeten die Gemeinde Bümpliz, die am 1. Januar 1919 als einzige Gemeinde von der Stadt Bern eingemeindet wurde (siehe Tab. 2 und Abb. 1).

Die Statistischen Bezirke variieren in der Fläche zwischen 10 ha (2 Weißes Quartier) und 1289 ha (30 Oberbottigen), bei der Einwohnerzahl 1976 zwischen 172 Personen (4 Gelbes Quartier) und 18852 Personen (29 Bümpliz) und bei der Beschäftigtenzahl 1975 zwi- schen 246 Personen (13 Weißenstein) und 18176 Personen (5 Rotes Quartier).

Für den Zweck der statistischen Mikroanalysen ergab sich deshalb die Notwendigkeit einer feineren Aufgliederung des Stadtgebietes. Leider wurde bei der Einteilung der Stadt Bern in die rund 1000 Zählkreise vorgängig der Eidgenössischen Volkszählung $1970 \mathrm{zu}$ wenig auf die Bedürfnisse einer späteren verfeinerten Datenauswertung geachtet, indem nur die herkömmlichen statistischen Grenzen, die Grenzen der Schulkreise und der Kirchgemeinden und die Verkehrsbezirke berücksichtigt, im übrigen aber die Zählkreise nur nach der Anzahl Haushalte gebildet wurden.

Da der Hektarraster in der Stadt Bern 1970 nicht angewendet wurde und für die Personen, Haushalte bzw. Wohnungen somit der Zählkreis die kleinste räumlich identifizierbare Einheit darstellt, hatte eine neue Aufgliederung des Stadtgebietes von der Zählkreiseinteilung auszugehen, $d . h$. die neu zu bildenden Einheiten mußten einen oder mehrere Zählkreise umfassen. Nach umfangreichen Studien und Probeauswertungen wurde die Feingliederung der Stadt Bern in "Quartiere» nach folgenden Regeln vorgenommen:

1) Die Wohnbevölkerung eines Quartiers soll zwischen etwa 300 und 1500 Personen liegen, wobei als Mittelwert 800 Personen angenommen werden.

2) Es sollen homogene Quartiere gebildet werden bezüglich baulicher Struktur, Alter und Nutzung der Bauten, soweit dies bei der vorgegebenen Zählkreiseinteilung und der beabsichtigten Quartiergröße möglich ist.

3) Die Quartiere müssen sich zu den Statistischen Bezirken und damit auch zu den Stadtteilen aufaddieren lassen.

4) Ein Quartier soll im allgemeinen ein zusammenhängendes Gebiet umfassen.

5) Große Kollektivhaushalte mit einseitiger Struktur (Spitäler, Heime, Anstalten, Schwesternhäuser) sollen nach Möglichkeit eine eigene Einheit bilden, um die Struktur der übrigen Bevölkerung nicht zu beeinflussen.

6) Große mehr oder weniger geschlossene Industriegebiete werden zu einem eigenen Quartier zusammengefaßt.

Dr. E. Gächter, Statistisches Amt der Stadt Bern, Gutenbergstraße 27, 3011 Bern. 
Tab. 1: Übersicht über die vorhandenen quartierweisen Daten (Stadt und Region Bern)

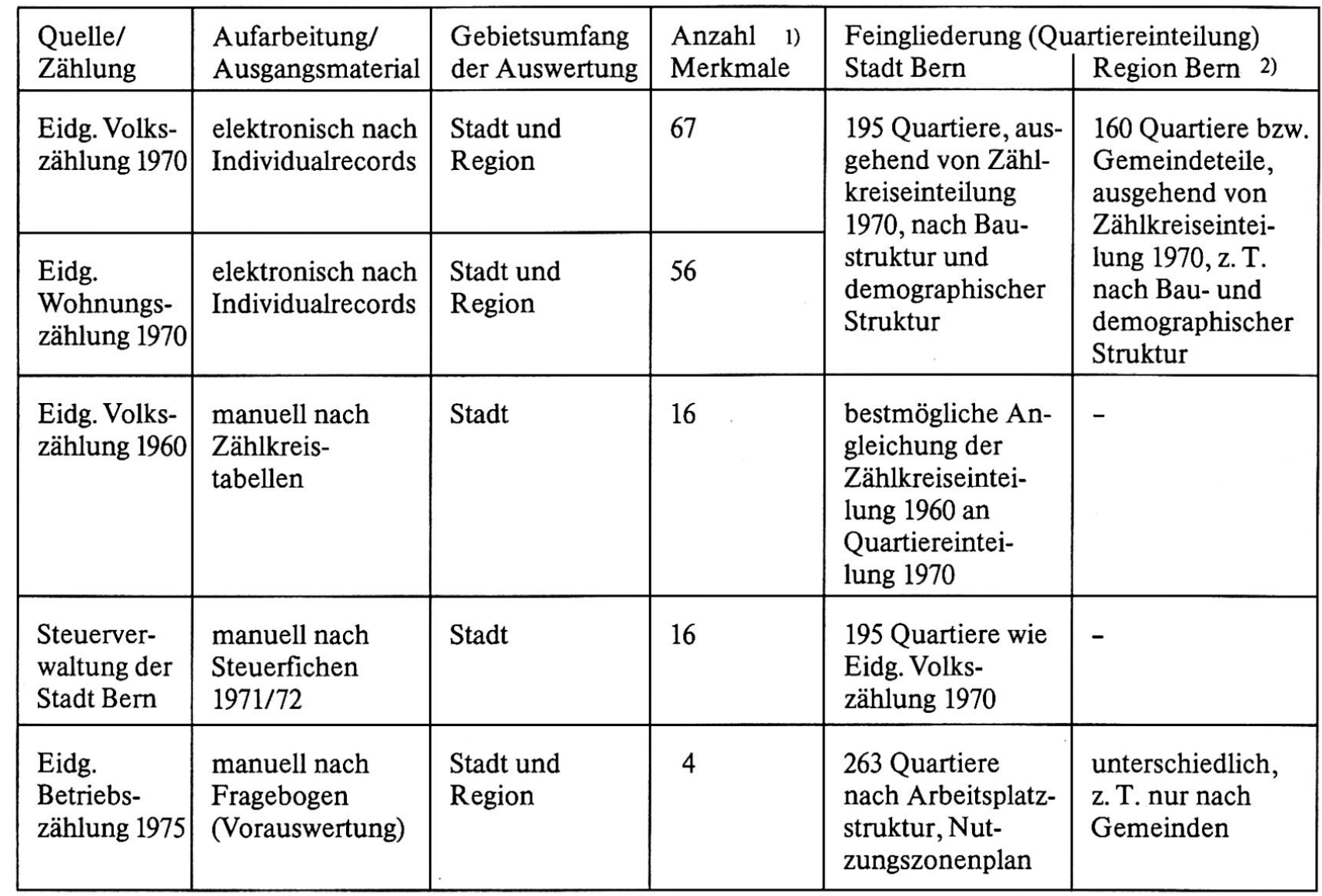

1) nur absolute Werte; ohne Merkmalskombinationen (Dichtewerte, relative Werte)

2) Gebiet des Regionalplanungsvereins Stadt Bern und umliegende Gemeinden, umfassend (Stand 1975) die Viertelsgemeinde Allmendingen, die Gemeinden Bäriswil, Bolligen (Viertelsgemeinden Bolligen, Ittigen, Ostermundigen), Bremgarten, Diemerswil, Frauenkappelen, Jegenstorf, Kehrsatz, Kirchlindach, Köniz, Mattstetten, Meikirch, Moosseedorf, Münchenbuchsee, Muri, Stettlen, Urtenen, Vechigen, Wohlen, Worb, Zollikofen (sowie die Stadt Bern)

Die Aufgliederung nach den angeführten Regeln erfolgte aufgrund von vorhandenem Quellenmaterial (z. B. GROSJEAN 1968) und von mehrwöchigen Struktur- und Nutzungserhebungen des Autors im Felde. In einigen Fällen scheiterte aber eine auf der Hand liegende Abgrenzung zwischen zwei unterschiedlich strukturierten Quartieren an der vorgegebenen Zählkreiseinteilung. Die Aussagekraft des Zahlenmaterials wurde dadurch natürlich eingeschränkt. Eine Beschreibung der 195 gebildeten Quartiere samt Karte ist in GÄCHTER 1974 I enthalten.

Vor der Eidgenössischen Betriebszählung 1975 stellte sich das Problem einer Feineinteilung erneut. Da aber Wohnbevölkerung und Arbeitsplätze unterschiedlich über die Stadt verteilt sind (Bezirk 4: 222 Einwohner, 7395 Arbeitsplätze, Bezirk 32: 14461 Einwohner, 3741 Arbeitsplätze 1975), kamen wir zur Überzeugung, daß für die beiden Großzählungen eine mindestens in einzelnen Gebieten unterschiedliche Einteilung notwendig sei. Unterdessen war auch der Nutzungszonenplan der Stadt Bern rechtskräftig geworden, der für die einzelnen Zonen bestimmte Parzellenund Gebäudenutzungen bzw. Mindestwohnanteile vorschreibt. Auf dieser Grundlage wurde in Zusammenarbeit mit dem Stadtplanungsamt Bern eine neue Einteilung festgelegt, die für verschiedene Zwecke dienen soll.

Die Stadt Bern wurde in einen Grundraster von 172 Einheiten gegliedert, der für beide Großzählungen angewendet werden soll. In Ergänzung dazu sind für die Volks- bzw. Betriebszählung weitere Aufteilungen vorgesehen, so daß sich 266 Volkszählungs- bzw. 217 Betriebszählungsquartiere ergeben. Als Gliederungskriterien dienten die schon für 1970 angewendeten Regeln (siehe oben) und der Nutzungszonenplan. Als minimale Grenzwerte für ein Quartier wurden 200 Einwohner bzw. 5 Arbeitsstätten mit total mindestens 100 Beschäftigten angenommen. Diese Grenzen gewährleisten einerseits die statistische Geheimhaltung von Individualdaten, andrerseits verhindern sie eine allzu große, unübersichtliche Anzahl von Einheiten. Die neue Aufgliederung der Stadt Bern wurde anläßlich der Eidgenössischen Betriebszählung vom September 1975 angewendet und soll auch anläßlich der Eidgenössischen Volkszählung 1980 wieder benützt werden. Unterdessen wurde sie auch der im 
Aufbau begriffenen Einwohner- bzw. Grundstückund Gebäude-Datenbank der Stadt Bern zugrunde gelegt.

\section{Die Bevölkerungs- und Wohnungsstruktur 1970}

\subsection{Allgemeines}

Die Stadt Bern weist wie alle Kernstädte der schweizerischen Großagglomerationen seit einigen Jahren eine rückläufige Wohnbevölkerung auf, während die umliegenden Regionsgemeinden bis etwa 1974 eine starke Bevölkerungszunahme verzeichneten.

Einer bedeutenden Bevölkerungsabnahme praktisch im ganzen Gebiet der fünf alten Stadtteile (Bezirke 1-28, siehe Abb. 2 und 3), verursacht durch Wohnraumverdrängung und Abnahme der Wohndichte (Personen pro Zimmer) im Altwohnungsbestand, steht nur noch eine Zunahme in den Gebieten mit starker Neubautätigkeit gegenüber: 1960-1970 Bezirke Bümpliz, Bethlehem, Schoßhalde (Überbauung Giacomettistraße) und 1970-1975 Bümpliz, Bethlehem, Murifeld (Überbauung Wittigkofen-West), Oberbottigen.

Ein Vergleich der quartierweisen Bevölkerungsentwicklung zwischen 1960 und 1970 ergab folgendes Bild: In 118 Quartieren (61\%) ging die Bevölkerung in den zehn Jahren um mehr als $5 \%$ zurück, in 89 Quartieren (46\%) sogar um mehr als 10\%. Extreme Bevölkerungsabnahmen zeigten z. B. das Rote Quartier $(-37,9 \%)$, die Quartiere Vordere Länggasse $(-33,3$ Prozent), Monbijou-Zentrum (-34,4\%), Weyermannshaus-Industrie $(-49,1 \%)$, Bethlehemacker I $(-32,6 \%)$. Es handelt sich bei den Quartieren mit Bevölkerungsverlusten um City- bzw. Cityrand- und Industriegebiete mit Wohnraumverdrängung, aber auch um reine Wohngebiete, wo sich durch Abnahme der Geburtenzahl und Wegzug von erwachsenen Kindern eine veränderte Bevölkerungs- und Altersstruktur ergibt.

Eine gleichbleibende bzw. zunehmende Bevölkerungszahl wiesen praktisch nur Quartiere mit größerer Neubautätigkeit zwischen 1960 und 1970 oder kurz vor 1960 auf. Rund 10000 der 1970 erfaßten 162405 Einwohner der Stadt Bern wohnten in 13 Quartieren, die zwischen 1960 und 1970 gänzlich neu überbaut worden waren.

Die quartierweise Datenauswertung der Volkszählung 1970 (siehe auch Tab. 1) umfaßte die folgenden Merkmalsgruppen: Wohnbevölkerung: Altersgliederung, Geschlecht, Nationalität, Muttersprache, Konfession, Bürgerort, Geburtsort, Wohnort vor fünf Jahren, abgeschlossene Schulbildung, Erwerbszugehörigkeit; Berufstätige: Erwerbsart (Wirtschaftssektor), Stellung im Beruf, Charakter der Unternehmung, Arbeitsort, benütztes Verkehrsmittel; Ausländer: Kategorie; Haushaltungen: Größe.

Aus dem Datenmaterial der Wohnungszählung 1970 wurden nach Quartieren aufgearbeitet: Bewohnte Gebäude: Geschoßzahl, andere Nutzung; besetzte Woh- nungen: Gebäudeart, Hauseigentümer, Besitzverhältnis, Wohnungsgröße, Bauperiode, Ausstattung mit Bad, Untermieteverhältnisse, Einzugsjahr der Bewohner, Mietpreise. Als Kombination zwischen Volksund Wohnungszählungsdaten berechneten wir den Anteil der Wohnungen mit Haushaltungsvorstand über 64 Jahren, die mittlere Anzahl Bewohner pro besetzte Wohnung und die mittlere Anzahl Bewohner pro Wohnraum nach Alter und Nationalität des Haushaltungsvorstandes.

Im weiteren wurden noch Daten aus der Eidg. Volkszählung 1960 und aus den Steuerfichen 1971/72 der Steuerverwaltung der Stadt Bern manuell nach Quartieren ausgewertet (siehe Tab. 1). Die umfangreichen Datentabellen sind in GÄCHTER 1974 I, GÄCHTER 1974 II und BUCHER/GÄCHTER 1975 publiziert.

Von den 195 Quartieren der Einteilung 1970 können 19 als Quartiere mit größerem Kollektivhaushalt, 8 als Quartiere mit vorherrschender Industrie und nur geringer Wohnbevölkerung und 3 als Quartiere mit einem ins Gewicht fallenden Anteil an landwirtschaftlicher Bevölkerung bezeichnet werden. Es verbleiben somit als reine oder unterschiedlich stark gemischte Wohnquartiere 165 Einheiten. Die Wohnbevölkerung der Quartiere schwankt zwischen 140 und 1746 Personen (Mittelwert 832,8), der größere Teil der Quartiere liegt zwischen 400 und 1100 Einwohnern.

Die verschiedenen ausgewerteten Merkmale weisen zwischen den Quartieren der Stadt recht beträchtliche Schwankungen auf, was an einigen Beispielen gezeigt werden soll (wobei nur die 165 «Wohnquartiere» berücksichtigt werden):

Der Anteil der Kinder unter 15 Jahren an der Gesamtbevölkerung des Quartiers schwankte zwischen 45,0\% (Bethlehemacker II) und 3,9\% (EffingerstraßeWest), der Anteil der Ausländer zwischen 37,8\% (Gelbes Quartier in der Innenstadt) und 1,4\% (WylergutEinfamilienhäuser). Im Quartier Eggimannstraße hatten $86,5 \%$ der erwachsenen Wohnbevölkerung nur Primarschulbildung, während im Quartier Unteres Murifeld-Einfamilienhäuser $46,9 \%$ der erwachsenen Wohnbevölkerung eine höhere Schulbildung (Gymnasium, Seminar, Technikum, Hochschule) aufwiesen. Obwohl Bern oft Beamtenstadt genannt wird, wiesen 1970 nur zwei Quartiere einen Anteil der Beschäftigten bei Bund, Kanton und Gemeinde von über $50 \%$ auf (gesamtstädtischer Durchschnitt 26,5\%). Das Reineinkommen pro Steuerpflichtigen schwankte zwischen Fr. 13 000.- (Lorraine-Nord) und Fr. 42 900.- (Hintere Schoßhalde) bei einem gesamtstädtischen Durchschnitt von Fr. 19700 .-.

Auch aus der Auswertung der Eidg. Wohnungszählung 1970 sollen noch einige extreme Quartierwerte angeführt werden: Den höchsten Anteil an vom Eigentümer selbst bewohnten Wohnungen zeigte das Quartier Wylergut-Einfamilienhäuser mit 84,3\%. Das Quartier Sulgeneck wies 60,0\% Einzimmerwohnungen auf, während im Quartier Oberes Murifeld 63,8\% der Wohnungen 5 und mehr Zimmer hatten. Die mittlere 


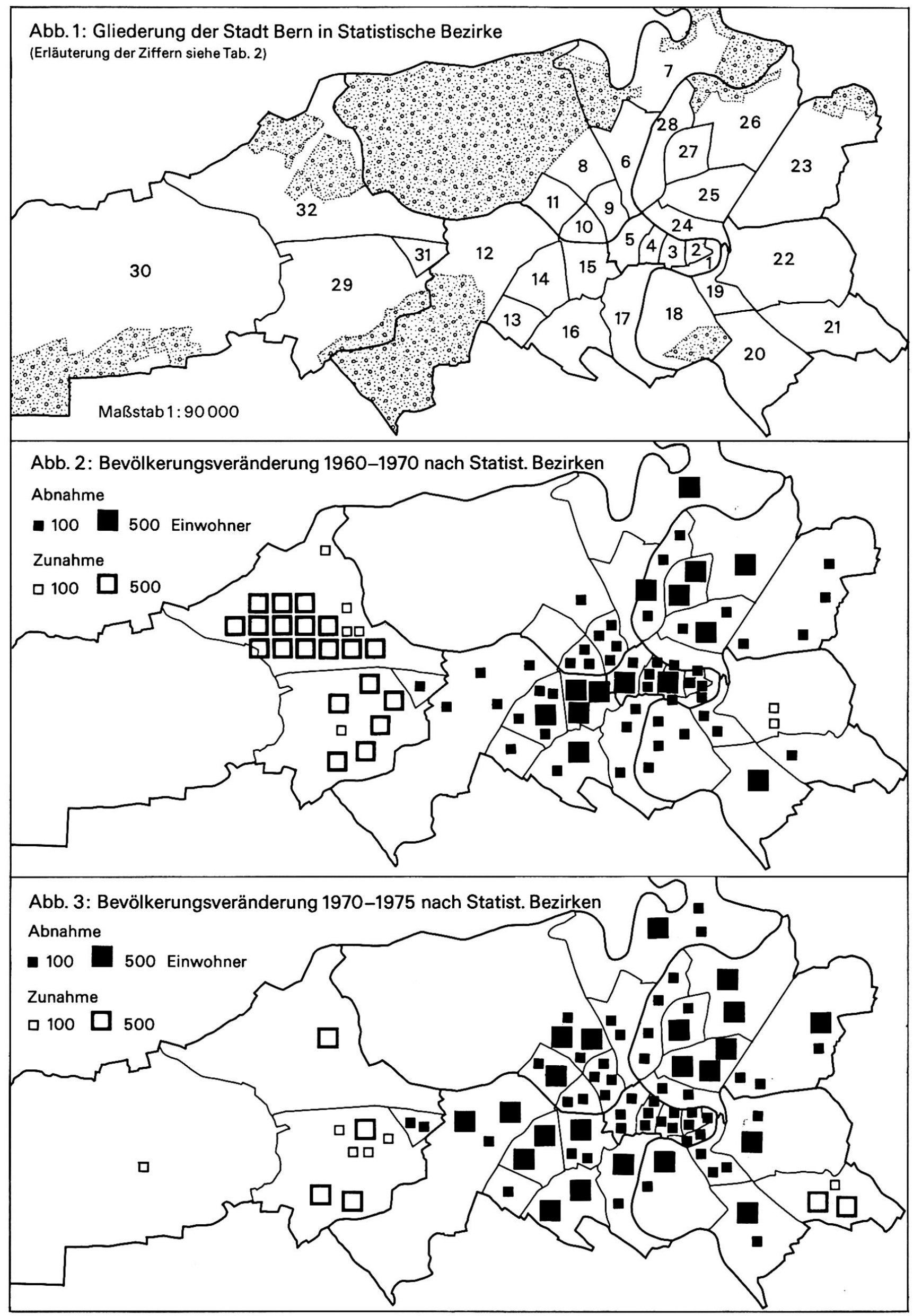


Tab. 2: Die Gliederung der Stadt Bern in Stadtteile und Statistische Bezirke

\section{Innere Stadt}

1 Schwarzes Quartier

2 Weißes Quartier

3 Grünes Quartier

4 Gelbes Quartier

5 Rotes Quartier

\section{Länggasse-Felsenau}

6 Engeried

7 Felsenau

8 Neufeld

9 Länggasse

10 Stadtbach

11 Muesmatt
III Mattenhof-WeiBenbühl

12 Holligen

13 Weißenstein

14 Mattenhof

15. Monbijou

16 Weißenbühl

17 Sandrain

IV Kirchenfeld-SchoBhalde

18 Kirchenfeld

19 Gryphenhübeli

20 Brunnadern

21 Murifeld

22 Schoßhalde

\section{$\checkmark$ Breitenrain-Lorraine}

23 Beundenfeld

24 Altenberg

25 Spitalacker

26 Breitfeld

27 Breitenrain

28 Lorraine

\section{Bümpliz-Oberbottigen}

29 Bümpliz

30 Oberbottigen

31 Stöckacker

32 Bethlehem

I (römische Zahlen): Stadtteile, 1 (arabische Zahlen): Statistische Bezirke

Anzahl an Zimmermieter weitervermietete Räume pro besetzte Wohnung erreichte im Quartier MonbijouOst den Wert von 0,51. Im Quartier Bethlehemacker I wurden $41,8 \%$ der Wohnungen von Bewohnern belegt, die vor 1947 einzogen. Der durchschnittliche monatliche Mietpreis für eine 3-Zimmerwohnung ohne Nebenkosten schwankte 1970 zwischen Fr. 164.- (Eggimannstraße) und Fr. 516.- (Giacomettistraße) bei einem städtischen Durchschnittswert von Fr. 284.-.

\subsection{Ausgewählte Quartiere}

Die quartierweisen Daten lassen sich in zwei Hauptrichtungen auswerten:

- gesamtstädtische Verbreitung eines Merkmals,

- Beschreibung eines Quartiers auf Grund der verschiedenen Merkmale.

Letztere Möglichkeit soll im folgenden am Beispiel von zwei Gegenüberstellungen von benachbarten Quartieren gezeigt werden.

Zunächst möchten wir zwei «historische» Quartiere vergleichen, nämlich den Südteil (GerechtigkeitsgasseSüdseite, Junkerngasse) und den Nordteil (Gerechtigkeitsgasse-Nordseite, Postgasse, Postgaßhalde) des Statistischen Bezirks 2 (Weißes Quartier, siehe Abb. 4 und 5). Rein äußerlich unterscheiden sich die beiden Quartiere schon dadurch, da $B$ das eine einen Teil des südlichen, sonnseitigen Abhangs der Aareschlaufe, das andere einen Teil des nördlichen, schattseitigen Abhangs umfaßt. Während das südliche Quartier durch den Straßennamen «Junkerngasse» schon charakterisiert wird bezüglich seiner früheren Bewohner, kann zum Nordteil gesagt werden, daß er vor der Stadterweiterung von 1256 das Metzgerviertel bildete und auch später ein Handwerker- und Gewerbequartier war.

Der Wohnanteil an der Bruttogeschoßfläche liegt bei rund 50 bis $70 \%$, nur $21 \%$ (Süd) bzw. $13 \%$ (Nord) der bewohnten Gebäude waren reine Wohngebäude (1970). Die Gebäude, meist 3- bis 6geschossig, standen zu rund $80 \%$ im Eigentum von Privatpersonen und wurden beinahe ausschließlich vor 1850 gebaut. Es herrschten Kleinwohnungen mit 1-3 Zimmern vor, im Südteil erreichten die Wohnungen mit 4 und mehr Zimmern $21 \%$, im Nordteil nur $12 \% .46 \%$ bzw. $40 \%$ der Bewohner von 1970 zogen erst zwischen 1966 und 1970 in die betr. Wohnungen ein, nur je etwas über $10 \%$ der Bewohner belegten schon vor 1947 dieselbe Wohnung. Die Wohndichte (Personen pro Wohnraum) betrug 0,68 (Süd) bzw. 0,83 (Nord). Die durchschnittlichen Mietpreise lagen im nördlichen Quartier deutlich unter denjenigen des südlichen Quartiers.

Wie sah nun die Bevölkerungsstruktur 1970 in den beiden Quartieren aus? Die Wohnbevölkerung umfaßte 683 bzw. 638 Personen (bei zwei Zahlenangaben bezieht sich die erste jeweils auf das südliche, die zweite auf das nördliche Quartier), der Ausländeranteil betrug $22 \%$ bzw. $26 \%$. In beiden Quartieren waren die Jugendlichen stark untervertreten gegenüber dem städtischen Durchschnitt, was sich auch in den hohen Erwerbsquoten von je $65 \%$ äußerte. Der Anteil der Stadtbürger an der schweizerischen Wohnbevölkerung betrug $13 \%$ bzw. $5 \%$ (städtischer Durchschnitt $6 \%$ ), je rund $75 \%$ der Bevölkerung wohnten bereits 1965 in der Stadt Bern. Im südlichen Quartier hatten $32 \%$ der Bevölkerung über 24 Jahren eine höhere Schulbildung, 34\% nur Primarschulbildung, im nördlichen Quartier lauteten die entsprechenden Werte dagegen $13 \%$ bzw. $62 \%$. Diese Relationen drücken sich auch in der beruflichen Stellung und im Einkommen aus: Anteil Direktoren und leitende Angestellte an den Erwerbstätigen $8 \%$ bzw. 3\%, Anteil anund ungelernte Arbeiter $30 \%$ bzw. $44 \%$. Reineinkommen pro Steuerpflichtigen Fr. 27 100.- bzw. Fr. 15 400.(städtischer Durchschnitt Fr. 19700.-). Es ergibt sich 


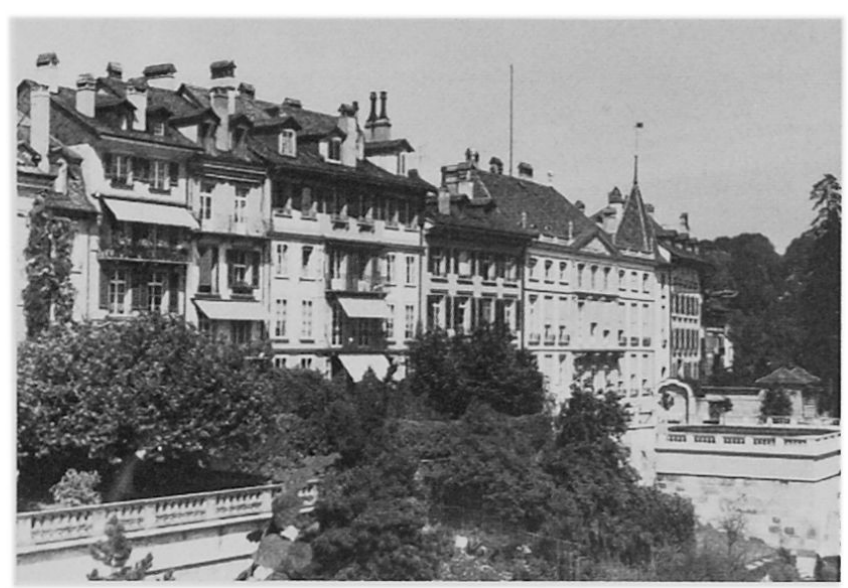

Abb. 4: Bern: Weißes Quartier-Süd. Gartenseitige Häuserfront der Junkerngasse von der Münsterplattform aus. Rechts außen Lerberhaus (Junkerngasse $43,1784 / 85$ ) und Erlacherhof (Junkerngasse 47, 1746-1752). Aufnahme Dr. E. Gächter, Sept. 1977

somit auch 1970 noch eine deutliche Differenzierung der beiden Teile des Statistischen Bezirks 2 (Weißes Quartier).

Die zweite Gegenüberstellung von Quartieren zeigt zwei für den Westen Berns und vermutlich auch andere Städte typische Quartierformen aus der Zeit nach 1940, nämlich die beiden Überbauungen Bethlehemacker I und II (siehe Titelbild; auf der Kartenbeilage Quartier der Gruppe 9 und 6 südöstlich des Wortes «Bethlehem»). Die Überbauung Bethlehemacker I wurde zwischen 1943 und 1948 von der Siedlungsgenossenschaft der Holzarbeiter-Zimmerleute des Schweizerischen Bau- und Holzarbeiterverbandes errichtet und befindet sich heute noch in deren Besitz. Die Siedlung umfaßte 1970152 Reiheneinfamilienhäuser zu drei bis fünf Zimmern mit 551 Einwohnern. Die Überbauung Bethlehemacker II wurde zwischen 1968 und 1974 nach Sonderbauvorschriften erstellt. Sie umfaßt drei Turmhochhäuser zu 22 Stockwerken (je rund 160 Wohnungen zu 1 bis 6 Zimmern) und zwei Scheibenhochhäuser mit 11 Stockwerken (rund 160 bzw. 200 Wohnungen mit vorwiegend 3 und 4 Zimmern). Die Häuser gehören z.T. ebenfalls der oben genannten und anderen Genossenschaften, z. T. Privatpersonen und Gesellschaften. Im Zeitpunkt der Volkszählung 1970 war erst ein Scheibenhaus (Haus rechts außen auf dem Titelfoto) bezogen, in dem 756 Personen wohnten. Ein Teil der Wohnungen des betreffenden Hauses sind Sozialwohnungen, d.h. von der öffentlichen Hand verbilligte Wohnungen für Familien mit niedrigem Einkommen.

Die beiden Quartiere weisen eine extrem unterschiedliche Baustruktur auf: Einfamilienhäuser/Hochhaus, 84 bzw. rund 600 Einwohner pro ha Quartierfläche. Trotzdem sind eine ganze Reihe demographischer Merkmale ähnlich gelagert (erste Zahl Einfamilienhausquartier, zweite Zahl Hochhaus): Geburtsort Stadt Bern 53,5/56,0\%, Anteil Personen über 24 Jahren mit nur Primarschulbildung 77,3/70,7\%, Anteil untere

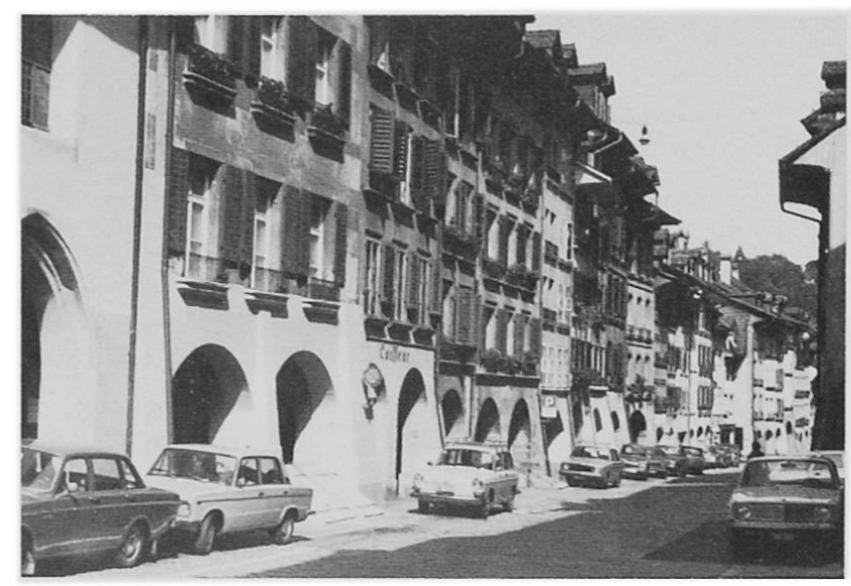

Abb. 5: Weißes Quartier-Nord. Nördliche Häuserreihe an der Postgasse, Blick gegen Osten. Links angeschnitten das Antonierhaus (Postgasse 62, ehemalige Kirche des Spitalordens der Antoniter, 1492-1505 erbaut). Aufnahme Dr. E. Gächter, Sept. 1977

Angestellte, Arbeiter an den Berufstätigen 85,1/92,9\%, Anteil Erwerbstätige in der Privatwirtschaft 78,5/77,7\%, Anteil Arbeitsort in Bethlehem 16,1/14,4\%, Anteil öffentliches Verkehrsmittel im Berufsverkehr 43,2/ $34,0 \%$. Das durchschnittliche Reineinkommen pro Steuerpflichtigen belief sich 1970 auf Fr. 15 200.- bzw. 14440.-. Diese Ähnlichkeit läßt sich daraus erklären, daß beide Quartiere den Typus des Arbeiterwohnquartiers - allerdings von 1945 bzw. 1970 - verkörpern.

Abweichend sind natürlich diejenigen Merkmale, die vor allem vom Alter der Bauten bzw. vom Einzugsjahr der Bewohner abhängen: Anteil Personen im Alter von 0-6 Jahren 6,5/28,3\%, Anteil der Erwerbstätigen an der Wohnbevölkerung 54,0/30,2\%. Die Mietpreise für eine Vierzimmerwohnung (ohne Nebenkosten) betrugen 1970 im Monat durchschnittlich Fr. 189.- (Einfamilienhaus) bzw. Fr. 362.- (Hochhaus). Mit diesen beiden Gegenüberstellungen zweier Quartiere sollte kurz gezeigt werden, welche Auswertungsmöglichkeiten mikrostatistischer Art die Großzählungen bieten.

\subsection{Versuch einer Quartiertypisierung mit quantitativen Methoden}

\subsection{Faktorenanalysen}

Bei der Fülle des quartierweisen Datenmaterials drängt sich eine quantitative Analyse auf. Diese ist jedoch mit einigen Schwierigkeiten verbunden, da sich nicht alle quartierweisen Daten auf die gleiche Grundgesamtheit beziehen: Die Volkszählungsangaben umfassen die gesamte Wohnbevölkerung (inkl. Personen in Kollektivhaushalten, aber exkl. Diplomaten), die Einkommenszahlen beziehen sich nur auf die in Bern steuerpflichtigen natürlichen Personen schweizerischer Nationalität (also ohne Wochenaufenthalter) und diejenigen Ausländer, die nicht der Quellensteuer 
unterliegen (d. h. die Niedergelassenen). Die Angaben aus der Eidgenössischen Wohnungszählung wiederum beziehen sich auf die Haushaltswohnungen (ohne Kollektivunterkünfte wie Fremdarbeiterbaracken, Altersheime, Schwesternhäuser). Aus diesen Gründen kamen die Quartiere mit bedeutenden Kollektivhaushalten und einzelne Industriequartiere mit Fremdarbeiterunterkünften für die Analyse nicht in Frage. Ebenso wurden die drei Quartiere mit einem höheren Anteil an landwirtschaftlicher Bevölkerung im äußersten Westen der Stadt weggelassen, da sie ein störendes Element in die Analyse städtischer Quartiere gebracht hätten.

Dazu kommt, daß viele Merkmale eine stark von einer Normalverteilung abweichende Wertstreuung haben. So weisen z. B. 106 der 195 Quartiere einen Wohnungsanteil mit eigenem Bad von über $95 \%$ auf bei einem städtischen Durchschnitt von $87,3 \%$ und einem Minimalwert von $5,0 \%$. Trotzdem wurden eine Reihe von Faktorenanalysen durchgeführt mit 165 bis 176 Quartieren und (programmbedingt) maximal 100 Variablen, die nicht transformiert wurden. Die Auswahl der Variablen wurde nach den folgenden Kriterien vorgenommen:

- nur für die demographische und bauliche Struktur wesentliche Variablen (nicht verwendet z. B. Bürgerort der Schweizer),

- nur relative und Dichtewerte,

- bei Variablen, die hundertprozentig oder nahezu hundertprozentig miteinander korreliert sind (z.B. Anteil Personen über 64 Jahren / Anteil Rentner, Pensionierte), wird nur eine Variable verwendet.

Die insgesamt sieben Analysenl) umfaßten z.T. nur demographische, nur wohnbauliche oder gemischt demographisch-wohnbauliche Variablen. Beim angewendeten Programm handelt es sich um eine Hauptkomponentenanalyse, ausgehend von einer Korrelationsmatrix der standardisierten Originaldaten (nähere Angaben siehe STEINER 1965).

Die Faktorenanalyse mit demographischen Variablen (inkl. Dichte- und Einkommensvariablen, total $54 \mathrm{Va}$ riablen) ergab folgendes Faktorenmuster: Der erste Faktor ist ein Niveaufaktor (Einkommen, Bildung, Stellung im Beruf), der $18,4 \%$ der Varianz der Ausgangsvariablen erklärt. 15 Variablen weisen eine Faktorenladung von 0,5 und mehr auf. Der zweite Faktor kann als Ausländerfaktor bezeichnet werden (erklärte Varianz 16,4\%, 13 Variablen mit Faktorenladung über 0,5). Der dritte Faktor läßt sich als Großhaushaltsfaktor interpretieren (erklärte Varianz 16,3\%, 13 Variablen mit Faktorenladung über 0,5). Als Neuzuzügerfaktor kann der vierte Faktor bezeichnet werden mit einer erklärten Varianz von $11,4 \%$ und 8 Variablen mit einer Ladung über 0,5 . Die weiteren Faktoren (insgesamt neun Faktoren mit Eigenvalue über 1,0) weisen eine deutlich geringere erklärte Varianz auf ( 7,2 bis $2,8 \%$ ) und sind $\mathrm{z}$. T. auch schwieriger zu interpretieren.
Die Faktorenanalyse mit baulichen Variablen umfaßte 22 Variablen. Sie ergab sechs Faktoren mit einem Eigenvalue über 1,0, die 78,3\% der gesamten Varianz der Ausgangsvariablen erklären. Der erste Faktor kann als Eigentümer- und Großwohnungsfaktor bezeichnet werden (erklärte Varianz 18,4\%, fünf Variablen mit Ladung über 0,5). Der zweite Faktor ist ein Mietpreisfaktor, der $15,8 \%$ der Gesamtvarianz erklärt und bei vier Variablen eine Faktorenladung über 0,5 aufweist. Ein Neubauten- und Hochhausfaktor ist der dritte extrahierte Faktor (erklärte Varianz 12,3\%, drei Variablen mit Ladung über 0,5). Der vierte Faktor ist mit einem einfachen Begriff nicht umschreibbar. Vier Variablen tragen hauptsächlich zu seiner Bildung bei (Anteil 2-Zimmerwohnungen [negative Ladung], Anteil 4-Zimmerwohnungen, Anzahl Bewohner pro Wohnung, Anteil Wohnungen mit Bad). Der fünfte Faktor kann als 1-Zimmerwohnungs- und Mischbautenfaktor, der sechste als Untermiete- und 4-6-Geschoß-HäuserFaktor bezeichnet werden.

Es muß hier noch angeführt werden, daß durch die Eidgenössische Gebäude- und Wohnungszählung die Wohnstruktur nur in gewissen Merkmalen erfaßt wird, während andere wichtige Aspekte (Wohnlage, Erschließung, Versorgung, Wohnqualität, Wohnkomfort, Wohnfläche der Wohnung, Bruttoausnützungsziffer der Parzelle) nicht berücksichtigt sind und deshalb das Ergebnis der Faktorenanalyse nur in beschränktem Maße das ganze Spektrum der Wohnungs- und Baustruktur wiedergeben kann. Für eine Verbesserung des Ergebnisses in dieser Hinsicht wäre die Erhebung von weiteren Merkmalen zusätzlich zur Eidgenössischen Wohnungszählung notwendig.

Das Hauptgewicht wurde auf die Analysen mit demographischen und baulichen Variablen gelegt. Die letzte der durchgerechneten Varianten soll hier näher geschildert werden: Sie umfaßte 65 Variablen und 165 Quartiere. Nur zwei Variablen erreichen in keinem der zehn extrahierten Faktoren mit einem Eigenvalue über 1,0 eine Faktorenladung über 0,5. Die ersten sechs Faktoren erklären $71,0 \%$, die zehn Faktoren $84,2 \%$ der Gesamtvarianz der Ausgangsvariablen.

Der erste Faktor ist (wie bei der rein demographischen Analyse) ein Niveaufaktor mit einer erklärten Varianz von $16,4 \%$ und 17 Variablen mit einer Faktorenladung über 0,5 (Schulbildung, Einkommen, Stellung im Beruf, Wirtschaftssektor, Wohndichte, Großwohnungen). Die Quartiere mit den höchsten Faktorenwerten liegen beinahe ausschließlich im Südosten der Stadt Bern (siehe auch Kartenbeilage), der niedrigste Wert ergibt sich für das Quartier Bethlehemacker I. Auch der zweite Faktor setzt sich ähnlich zusammen wie bei der demographischen Analyse (Ausländerfaktor, erklärte Varianz 15,5\%, 16 Variablen mit Ladung über 0,5: Ausländeranteil, Wohnort vor fünf Jahren, Muttersprache, Anteil Erwerbstätige, Konfession, Stellung im Beruf u. a.). Die Faktorenwerte der Quartiere sind ziemlich einseitig verteilt, d.h. einer kleinen Zahl von Quartieren mit extrem hohen Werten (City-Gebiete u. a.) 
stehen eine große Zahl von Quartieren mit durchschnittlichen bis leicht unterdurchschnittlichen Werten gegenüber.

Als dritter Faktor wurde ein Neubauten-Neuzuzügerfaktor extrahiert (erklärte Varianz 12,5\%, 11 Variablen mit hoher Ladung: Einzugsjahr, Baujahr, Wohnort vor fünf Jahren, Anteil 0-6jährige Personen, Geschoßzahl). Mit hohen Faktorenwerten erscheinen hier die bekannten Großüberbauungen aus jüngster Zeit. Auf der anderen Seite der Skala liegen aber nicht «Altbauquartiere», sondern vorwiegend Gebiete, die in den fünfziger Jahren überbaut wurden. Der vierte Faktor kann als Großhaushaltfaktor bezeichnet werden. Er erklärt $12,2 \%$ der Gesamtvarianz und 11 Variablen weisen eine Faktorenladung über 0,5 auf (Bewohner pro Wohnung, Haushaltsgröße, Altersstruktur, Wohnungsgröße, Geburtsort). Hohe Faktorenwerte finden wir vor allem im Westteil der Stadt (Bümpliz, Bethlehem). Der fünfte Faktor läßt sich als Einfamilienhaus- und Eigentümerwohnungsfaktor interpretieren (erklärte Varianz 7,6\%, Variablen mit hohen Ladungen: Geschoßzahl, Gebäudeart, Besitzverhältnis der Wohnungen, Bevölkerungsdichte [negativ], Wohnungsgröße). Die höchsten Faktorenwerte werden in den ArbeiterEinfamilienhausquartieren und in Gebieten im Südosten der Stadt Bern erreicht. Der sechste Faktor, der gebildet wird, kann als Mittelschichtfaktor bezeichnet werden. Die erklärte Varianz liegt noch bei $6,8 \%$, fünf Variablen weisen Faktorenladungen über 0,5 auf (Einkommen, Einkommensstreuung, Stellung im Beruf, Altersstruktur, Wohnungen mit Bad). Die Quartiere mit hohen und niedrigen Faktorenwerten sind hier stark über die ganze Stadt gestreut.

Die weiteren extrahierten Faktoren weisen nur noch eine geringere Bedeutung auf (erklärte Varianz zwischen 5,8 und 2,3\%). Sie können als Altbau-Überalterungsfaktor, Kleinwohnungsfaktor und Faktor der 3-4geschossigen Wohnbauten bezeichnet werden. Der zehnte Faktor schließlich wird vorwiegend aus den beiden Variablen Einzugsjahr 1960-1965 und Arbeitgeber Privatwirtschaft gebildet.

Die Faktorenanalysen ergaben somit ein interessantes Ergebnis. Die von den extrahierten Faktoren erklärte Varianz ist jeweils recht groß, ohne daß ein Faktor allzusehr dominiert. Vielmehr resultiert eine ganze Reihe von praktisch gleichwertigen Faktoren. Durch eine homogenere Quartiereinteilung, eine bessere Abtrennung der Kollektivhaushalte und zusätzliche Merkmale bei der wohnbaulichen Struktur könnte aber vermutlich das Ergebnis noch verbessert werden.

\subsection{Cluster-Analysen (Gruppenbildung)}

Anschließend an die Faktorenanalysen wurde versucht, eine Typisierung der Quartiere und eine Gruppenbildung durchzuführen. Dazu wurde ein Cluster-AnalyseProgramm verwendet, das auf dem Prinzip der mehrdimensionalen Distanzgruppierung beruht. Als Input dienten die Faktorenwerte der zuletzt beschriebenen
Analyse (165 Quartiere, Faktoren 1-6; Faktor 1 bis 5 mit 1,5 gewichtet, Faktor 6 ungewichtet).

Bedingt durch die fehlende Normalverteilung bei vielen Variablen ergab sich, wie wir schon gesehen haben, mindestens bei einzelnen Faktoren eine ungleiche Verteilung der Faktorenwerte. Damit war zu erwarten, $\mathrm{da} ß$ sich dieser Umstand auch in der Cluster-Analyse auswirken würde.

Der Aufspaltungsstammbaum zeigte dann auch eine spezielle, stark einseitige Form. Nach den ersten zwölf Aufspaltungen resultierte ein großer Block von 135 Quartieren, daneben 10 Gruppen mit 2 bis 6 Quartieren und 2 Einzelquartiere. Nach 22 Schritten war immer noch ein Block von 73 Quartieren beisammen, während die anderen 92 Quartiere sich schon in 22 Gruppen von 1 bis 24 Quartieren aufgliederten.

Die Gruppenbildung weicht somit vom Bild, wie es z. B. in KILCHENMANN/GÄCHTER 1969 beschrieben ist, stark ab. Dies läßt sich daraus erklären, daß im mehrdimensionalen Faktorenraum eine große Zahl von Punkten im Bereich des Nullpunktes angehäuft ist und kleinere Gruppen oder Einzelquartiere in verschiedenen Richtungen und z. T. größeren Abständen lokalisiert sind. Da die Distanzen gegen außen immer größer werden, werden zunächst diese Punkte einzeln oder in kleinen Gruppen vom Gros abgespaltet bzw. im umgekehrten Betrachtungssinne zuletzt zusammengefaßt, während eine große Punktwolke lange undifferenziert bleibt, bzw. schon früh zusammengefaßt wird. Das Ergebnis der Cluster-Analyse ist unbefriedigend und für den gewünschten Zweck nur bedingt brauchbar.

Es wurde deshalb versucht, ausgehend von den quantitativen Ergebnissen der Cluster-Analyse, aber auch unter Berücksichtigung von weiteren, mehr qualitativen Gesichtspunkten, eine Um- bzw. Neugruppierung vorzunehmen. Dazu wurden in der gleichen Richtung im Faktorenraum liegende Gruppen oder Einzelquartiere (qualitativ ähnliches Faktorenmuster), die wegen der großen Distanz untereinander in einem Faktor - in der quantitativen Analyse nicht zusammengefaßt werden, in eine Gruppe zusammengenommen. Als Resultat ergaben sich vierzehn Gruppen, wie sie in der Kartenbeilage und in Tab. 3 dargestellt sind. Die Reihenfolge in der Karte und der Tabelle ist dabei nicht etwa durch die Reihenfolge bei der Aufspaltung, sondern durch die durchschnittlichen Faktorenwerte in den Faktoren 1 bis 6 (positive Extremwerte) bestimmt. Die Faktorenwerte werden in der Tabelle nicht in exakten Zahlen wiedergegeben, sondern in sieben Gruppen zusammengefaßt $(+++$ bis $---)$.

Im weiteren wurden noch elf Quartiere, die in den quantitativen Analysen weggelassen werden mußten (Industriegebiete mit bewohntem Teil, Wohnquartier mit größerem Kollektivhaushalt, siehe Abschnitt 3.31), auf Grund ihrer Werte in einzelnen Variablen den 14 Gruppen zugeteilt und die bewohnten Quartierteile in der Karte entsprechend angefärbt. 
Tab. 3: Faktorenwerte der Quartiergruppen (Demographisch-bauliche Struktur der Stadt Bern 1970, Erläuterung zu Kartenbeilage)

\begin{tabular}{|c|c|c|c|c|c|c|c|c|c|c|c|c|c|c|}
\hline Gruppe (Nr. auf Kartenbeilage) & 1 & 2 & 3 & 4 & 5 & 6 & 7 & 8 & 9 & 10 & 11 & 12 & 13 & 14 \\
\hline Anzahl Quartiere & 5 & 28 & 7 & 6 & 4 & 9 & 13 & 11 & 9 & 15 & 12 & 18 & 15 & 23 \\
\hline $\begin{array}{l}\text { 1. Faktor } \\
\text { (Niveaufaktor) }\end{array}$ & +++ & ++ & $\begin{array}{c}0 \\
1-\text { bis }++1\end{array}$ & + & + & - & - & O & -- & - & O & + & - & - \\
\hline $\begin{array}{l}\text { 2. Faktor } \\
\text { (Ausländerfaktor) }\end{array}$ & $\begin{array}{c}++ \\
1-\text { bis }++1\end{array}$ & $\begin{array}{c}- \\
1-- \text { bis }++1\end{array}$ & +++ & $\begin{array}{c}+++ \\
1++b .+++1\end{array}$ & $\begin{array}{c}+ \\
\text { lobis }++1\end{array}$ & $\begin{array}{c}- \\
1-\text { bis }+1\end{array}$ & 0 & - & $\begin{array}{c}- \\
(-- \text { bis }+++1)\end{array}$ & - & + & - & $\begin{array}{c}0 \\
1-- \text { bis }++1\end{array}$ & O \\
\hline $\begin{array}{l}\text { 3. Faktor } \\
\text { (Neubauten- und } \\
\text { Neuzuzügerfaktor) }\end{array}$ & - & $\begin{array}{c}0 \\
1-- \text { bis }++1\end{array}$ & 0 & - & +++ & $\begin{array}{c}+++ \\
1+\text { bis }+++1\end{array} \mid$ & - & -- & - & - & + & - & O & 0 \\
\hline $\begin{array}{l}\text { 4. Faktor } \\
\text { (Großhaushaltsfaktor) }\end{array}$ & + & 0 & - & + & -- & $\begin{array}{c}++ \\
\text { lobis }+++1\end{array}$ & $\begin{array}{c}++ \\
\text { lobis }+++ \text { ) }\end{array}$ & + & $\begin{array}{c}+ \\
\mid- \text {-bis }+++1\end{array}$ & $\begin{array}{c}+ \\
\text { 1-bis }++1\end{array}$ & - & - & -- & - \\
\hline $\begin{array}{l}\text { 5. Faktor } \\
\text { (Einfamilienhaus- und } \\
\text { Eigentümerwohnungsfaktor) }\end{array}$ & ++ & $\begin{array}{c}+ \\
1-\text { bis }+++1\end{array}$ & - & - & + & $\begin{array}{c}- \\
1-- \text { bis }+1\end{array}$ & - & - & $\begin{array}{c}+++ \\
(+ \text { bis }+++1\end{array}$ & $\begin{array}{c}+ \\
\text { (-bis }++1\end{array}$ & $\begin{array}{c}- \\
\text { (-bis }+1\end{array}$ & - & 0 & - \\
\hline $\begin{array}{l}\text { 6. Faktor } \\
\text { (Mittelschichtfaktor) }\end{array}$ & $\begin{array}{c}++ \\
\text { (-bis }+++1\end{array}$ & $\begin{array}{c}- \\
1---b i s+1\end{array}$ & $\begin{array}{c}- \\
(---b i s+1\end{array}$ & + & ++ & $\begin{array}{c}0 \\
1-- \text { bis }++1\end{array}$ & + & ++ & $\begin{array}{c}0 \\
1-- \text { bis }++1\end{array}$ & $\begin{array}{c}0 \\
1-- \text { bis }++1\end{array}$ & $\begin{array}{c}+ \\
1+\text { bis }++1\end{array}$ & $\begin{array}{c}+ \\
1+\text { bis }++1\end{array}$ & $\begin{array}{c}-- \\
(--- \text { bis ol }\end{array}$ & 0 \\
\hline
\end{tabular}

$\begin{array}{ll}\text { Faktorenwerte: } & +++: \text { über } 2,0,++: 1,001 \text { bis } 2,0,+: 0,251 \text { bis } 1,0,0:-0,25 \text { bis } 0,25,--- \text { : unter }-2,0,--:-1,001 \text { bis }-2,0,-:-0,251 \text { bis }-1,0 \\ \text { (ungewichtete Werte) }\end{array}$

Angegeben ist der mittlere Faktorenwert der Gruppe. Wo der Faktorenwert eines Quartiers um mehr als 1,0 vom Gruppenmittel abweicht, ist die maximale Streuung in Klammern beigefügt. 
Abb. 6: Gruppenbildung: Graphische Darstellung der Gruppenräume (ausgewählte Gruppen, siehe Kartenbeilage und Tab. 3; Faktorenwerte ungewichtet)

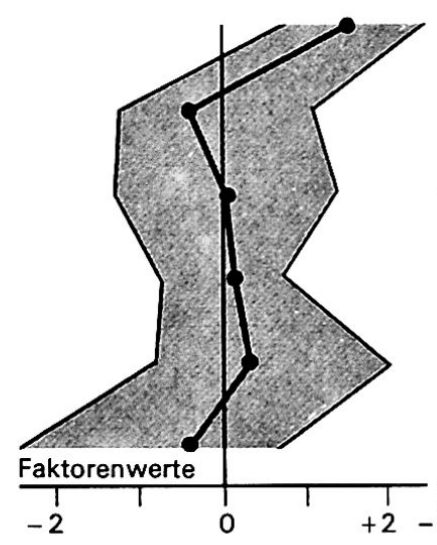

Gruppe 2 (24 Quartiere)

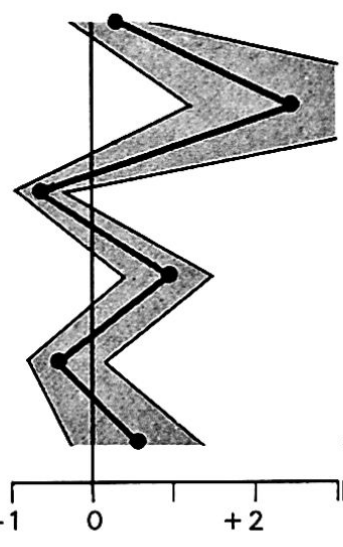

Gruppe 4 (6 Quart.)

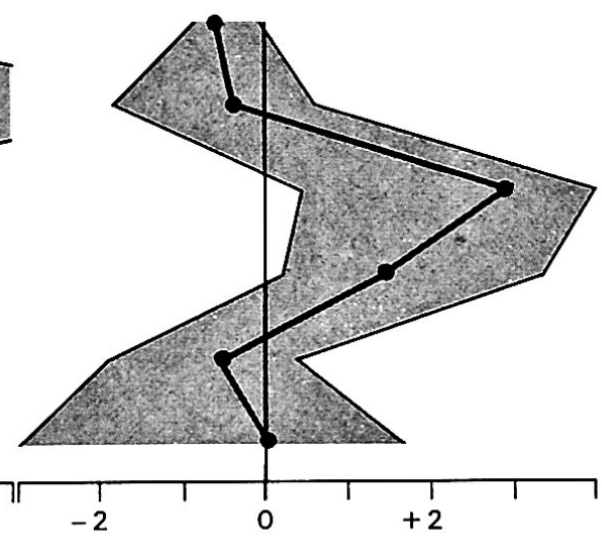

Gruppe 6 (9 Quartiere)

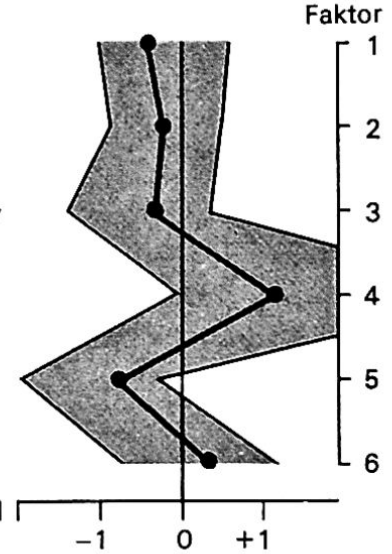

Gruppe 7 (13 Quart.)

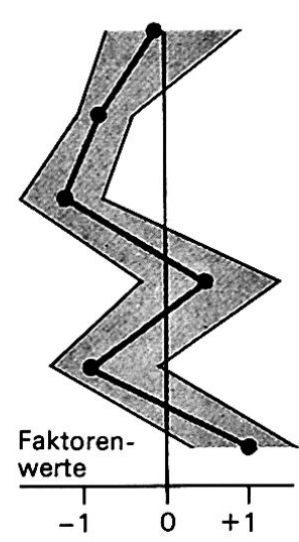

Gruppe 8 (10 Q)

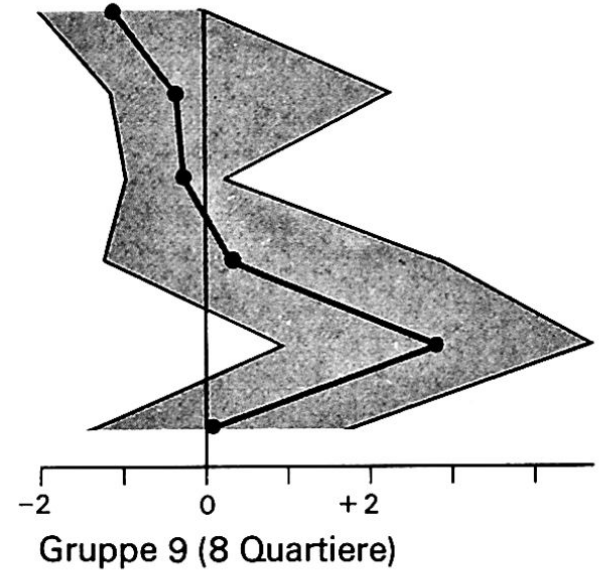

Gruppe 9 (8 Quartiere)

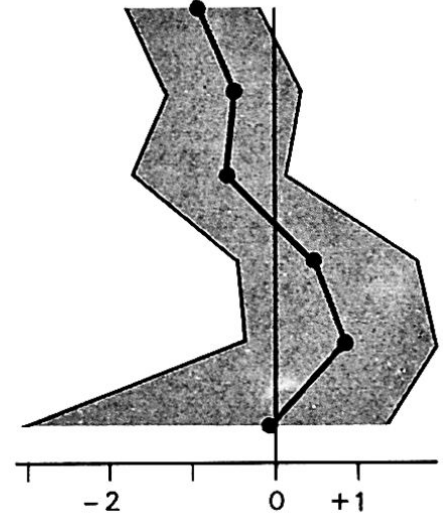

Gruppe 10 (15 Quartiere)

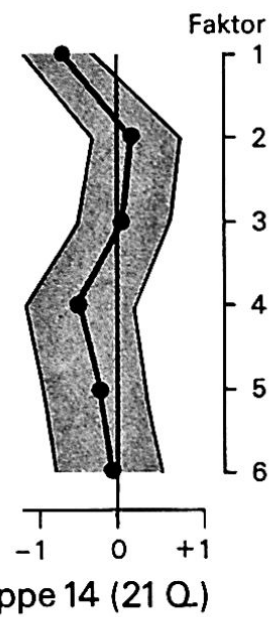

Im folgenden sollen nun die vierzehn Gruppen kurz charakterisiert werden: Die Gruppe 1 fällt durch die hohen positiven Werte in mehreren Faktoren auf. Speziell hervorzuheben ist das hohe Einkommensund Sozialniveau bei gleichzeitig hohem Ausländerbestand, was sich durch den relativ hohen Anteil an ausländischen und internationalen Beamten unter den Ausländern erklärt. Die fünf Quartiere liegen in den Gebieten Kirchenfeld-Brunnadern und Villette.

Die Gruppe 2 wird durch ein hohes Einkommensund Sozialniveau bei einem (bis auf Ausnahmen) niedrigen Ausländerbestand charakterisiert. Eher über dem Durchschnitt liegt auch der fünfte Faktor (Einfamilienhäuser, Eigentümerwohnungen). Wie Abb. 6 zeigt, ist die Streuungsbreite der Faktorenwerte relativ groß. Die Gruppe zählt 28 Quartiere2), die vorwiegend im Südosten der Stadt (Kirchenfeld-Schoßhalde) bzw. ringförmig außerhalb des früheren Befestigungsgürtels («Landhäuser, Landgüten»; vordere Länggasse, Altenberg, Obstberg usw.) zu finden sind.

In der dritten Gruppe sind sieben Quartiere mit einem hohen Wert im Ausländerfaktor, sonst aber durchschnittlichen bis unterdurchschnittlichen Faktorenwerten zu finden. Es handelt sich um drei Gebiete (untere Altstadt, Sulgenbach und Linde) mit einem mindestens z.T. renovationsbedürftigen Altbaubestand, der zu einer Konzentration von ausländischen Bewohnern geführt hat.

Die Gruppe 4 umfaßt sechs Quartiere mit extrem hohem Wert im 2. Faktor (hoher Ausländerbestand, hohe Bevölkerungsmobilität, hoher Anteil Wohnungen in gemischt genutzten Gebäuden, hoher Anteil 16- bis 24jährige Personen), die im Stadtzentrum oder in Kernzonen von Stadtteilen (Bümpliz, Spitalacker) liegen. Die Streuungsbreite der Faktorenwerte (Abb.6) ist dabei recht klein.

Die vier Quartiere der fünften Gruppe können als Neubauquartiere mit hohen Mietpreisen und deshalb eher überdurchschnittlichen Einkommen und kleineren Haushalten charakterisiert werden. Es handelt sich um die Überbauungen Waldheim, Fischermätteli, Sulgeneck und Giacomettistraße.

Die neun Quartiere der Gruppe 6 liegen alle im Westteil der Stadt und umfassen große Neuüberbauungen mit z. T. Sozialwohnungen (unterdurchschnittliche Einkommen, große Familien, relativ geringer Ausländeranteil): Tscharnergut, Gäbelbach, Bethlehemacker II, Schwabgut u. a. Wie Abb. 6 zeigt, ist die 
Variationsbreite innerhalb der Gruppe recht groß, obwohl es sich nur um neun Quartiere handelt.

In der Gruppe 7 werden dreizehn Quartiere zusammengefaßt, die mehrheitlich in den Jahren 1947-1960 überbaut wurden. Sie fallen auf durch relativ große Haushalte und eine hohe Wohndichte (Faktor 4), sind sonst aber eher durchschnittlich bis unterdurchschnittlich in den Faktorenwerten. Wir finden sie vor allem im Westen der Stadt.

Die Gruppe 8 liegt relativ nahe bei der Gruppe 7. Sie unterscheidet sich u.a. durch negativere Werte im 3. Faktor. Bei den elf Quartieren handelt es sich beinahe ausschließlich um Reihenmehrfamilienhaussiedlungen von 3 bis 4 Stockwerken, die zwischen 1947 und 1960 erbaut wurden. Zwei größere Flächen derartiger Quartiere sind in Holligen und im Breitfeld anzutreffen.

Die Gruppe 9 ist durch den stark negativen Wert im Niveaufaktor und den hohen Wert im Einfamilienhausfaktor gekennzeichnet. Es handelt sich um die ArbeiterEinfamilienhaussiedlungen, wie wir im Quartier Bethlehemacker I bereits ein Beispiel gesehen haben. Die neun Quartiere zeichnen sich auch noch durch ihre beinahe durchwegs periphere Lage aus. Wie Abb. 6 zeigt, ist die Streuungsbreite der Faktorenwerte recht groß, vor allem im 2. Faktor.

Nahe verwandt mit der vorhergehenden Gruppe ist die Gruppe 10. Sie unterscheidet sich vor allem durch niedrigere Werte beim 2. und 5. Faktor (Abb. 6). Die fünfzehn Quartiere, die vor allem im Westen und Norden der Stadt lokalisiert sind, zeichnen sich durch kleinere, meist einzeln stehende Mehrfamilienhäuser aus der Zeit von etwa 1920 bis 1950 aus.

Bei den Gruppen 11 bis 14 handelt es sich um diejenigen Quartiere, die - zusammen mit einem Teil der Gruppe 7 - in der Cluster-Analyse den großen, nach 22 Schritten noch nicht aufgespaltenen Block bildeten. Die Faktorenwerte weichen deshalb nur wenig von null ab und die Gruppen sind schwer zu charakterisieren. Die Mehrzahl der Quartiere dieser Gruppen liegen in den drei Stadtteilen Länggasse, MattenhofWeißenbühl und Breitenrain-Lorraine.

Die Gruppe 11 zeichnet sich durch eher positive Werte in den Faktoren 2, 3 und 6 aus. In der Gruppe 12 weist der Niveaufaktor einen überdurchschnittlichen Wert auf bei sonst beinahe durchwegs leicht negativen Faktorenwerten. Die Gruppe zeigt eine gewisse Ähnlichkeit zur Gruppe 2, die Quartiere liegen vielfach auch in der Nähe von Quartieren dieser Gruppe.

Die Gruppe 13 wird durch deutlich negative Werte bei den Faktoren 4 (Großhaushalte) und 6 (Mittelschicht) bei sonst durchschnittlichen bis leicht negativen Werten charakterisiert. In einzelnen Fällen hat der Ausländerfaktor noch ein größeres positives Gewicht. Damit ist eine gewisse Verwandschaft zur Gruppe 3 vorhanden. Die Quartiere der Gruppe 13 sind vor allem im Gebiet Lorraine-Breitenrain und südlich der Altstadt (Marzili-Matte) zu finden.

Die Gruppe 14 schließlich wird durch 23 Quartiere ge- bildet, die sich in den Gebieten Breitenrainplatz, Mattenhof und Länggasse konzentrieren. Die Gebäude (vielfach 4-6geschossige Häuser in geschlossener Bauweise) stammen zum größten Teil aus der Periode vor 1910. Wie Abb. 6 zeigt, weist die große Gruppe nur eine schmale Variationsbreite in den Faktorenwerten auf, die zudem alle nahe beim Mittelwert liegen. Wie schon bei der Beurteilung der Ergebnisse der Faktorenanalyse festgestellt wurde, weist der vorliegende Raumgliederungsversuch gewisse Unzulänglichkeiten auf. Er ist aber trotzdem für die Betrachtung der Probleme der kleinräumigen Stadtstruktur und -entwicklung ein nützliches Hilfsmittel.

\subsection{Karte der Quartierstruktur 1970}

Es seien an dieser Stelle noch einige mehr technische Bemerkungen zur farbigen Kartenbeilage erlaubt.

Die Karte stellt, wie auch die Abb. 1 bis 3 und 7 bis 11, nicht das ganze Stadtgebiet dar. Bedingt durch die Flächenausdehnung (maximale Länge Ost-West $15 \mathrm{~km}$, Nord-Süd 7,5 km) wird im Westen je ein unterschiedlich großes Stück weggelassen. Da es sich aber beim Bezirk Oberbottigen um ein vorwiegend land- und forstwirtschaftlich genutztes Gebiet handelt (1970: 1050 Einwohner auf 12,9 km² Fläche) und die drei betreffenden Quartiere nicht in die Analysen einbezogen wurden, spielt dieser Umstand keine große Rolle.

Die Farben für die vierzehn Gruppen wurden nach folgenden Kriterien ausgewählt: In gelben Farbtönen sind die Gruppen 1 und 2 dargestellt (hohe Werte in Faktor 1). Mit blauen Tönen ausgemalt sind die Quartiere der Gruppen 3 und 4 (hohe Werte im Ausländerfaktor). Rote Farben bezeichnen die Neubauquartiere nach 1960 (Gruppe 5 und 6), während die orange und violett ausgemalten Quartiere (Gruppe 7 und 8) vorwiegend zwischen 1947 und 1960 überbaut wurden und sich durch hohe Werte in den Faktoren 4 und 6 auszeichnen.

In grünen Farbtönen sind die Gruppen 9 und 10 dargestellt. Sie zeichnen sich durch hohe Werte in den Faktoren 4 und 5 aus bei sonst eher negativen Werten. Mit grauen und braunen Farben wurden schließlich die Gruppen 11 und 14 dargestellt.

Neben den farbigen Quartierflächen sind noch drei weitere Nutzungsformen durch schwarze Signaturen unterschieden: Industriegebiete, Lagerhäuser, Verkehrsbauten u. ä. sind durch eine waagrechte Flächenschraffur gekennzeichnet. Durch Einzelsignaturen werden bedeutende öffentliche und halböffentliche Gebäude dargestellt, wobei in unbewohnte (Kreissignatur; Verwaltungsgebäude, Schulen, Kirchen usw.) und bewohnte Gebäude (Dreiecksignatur; Spitäler, Heime, Anstalten u. ä.) unterschieden wird.

Die Karte wurde im Original mit schwarzer Tusche und Farbstiften auf einem Graudruck der Landeskarte $1: 25000$ (Situation, Schrift und Waldraster) gezeichnet. In einem elektronischen Scanner der Firma 


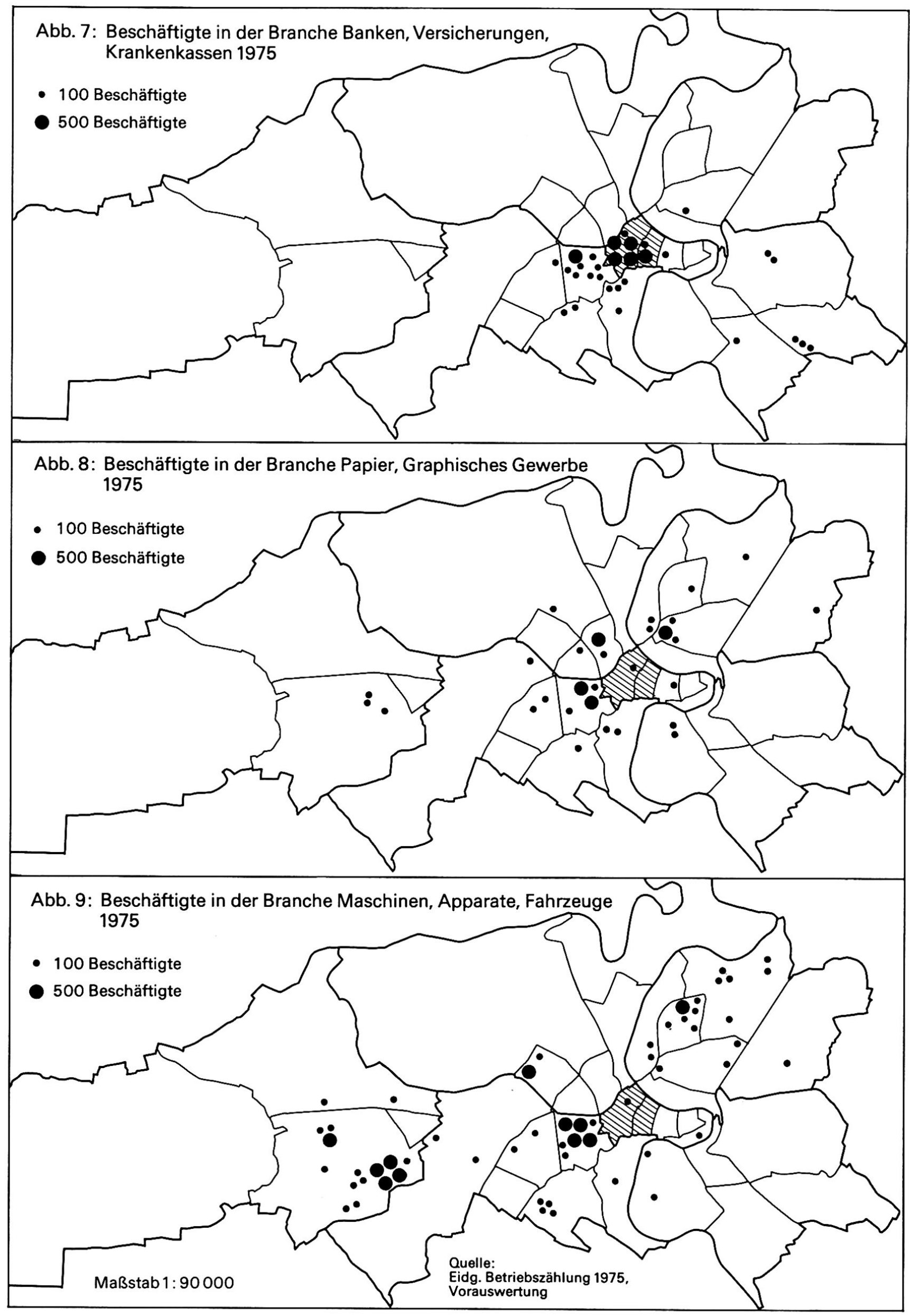




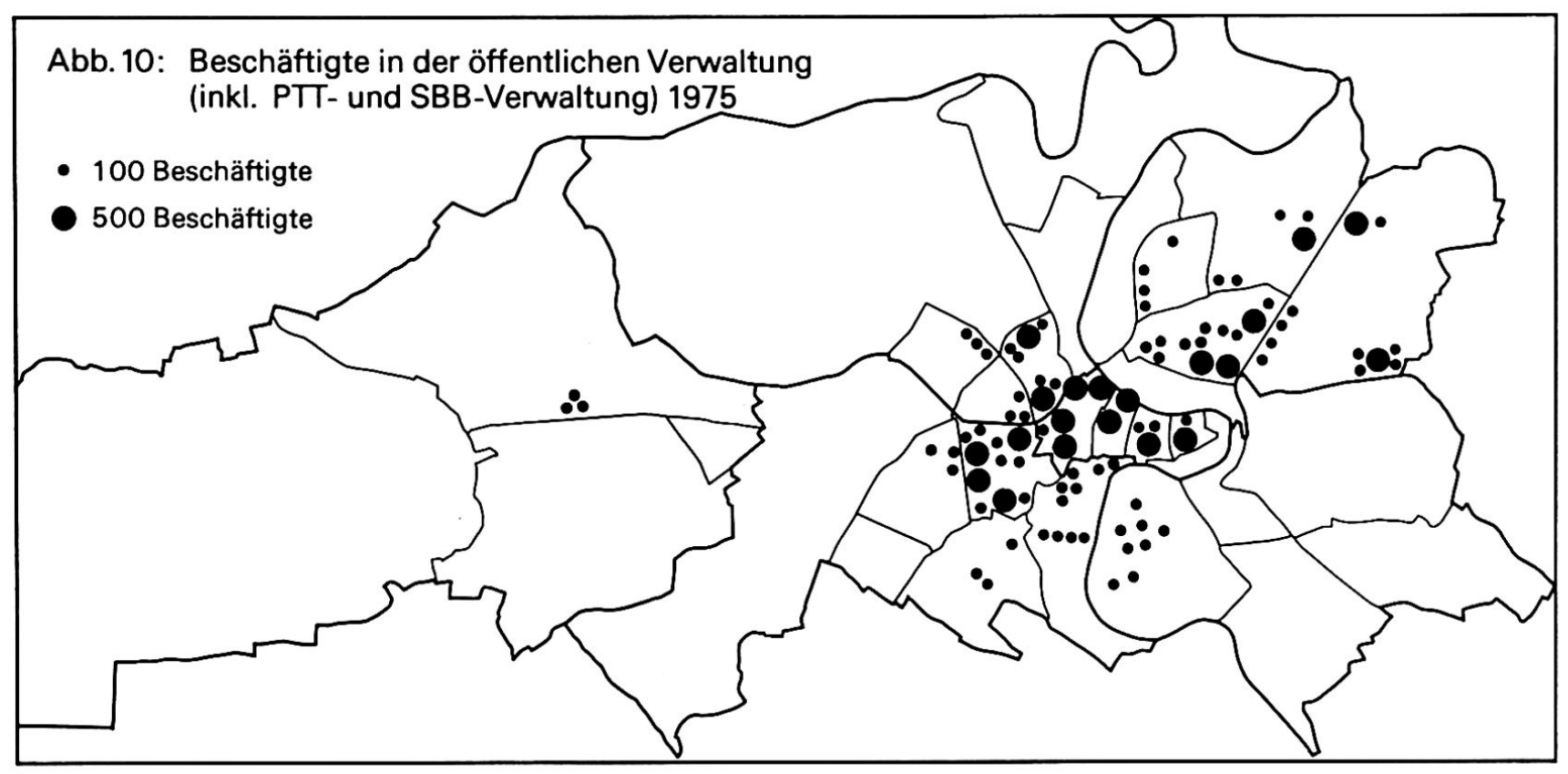

Dr.-Ing. R. Hell, Kiel (Details siehe RAETH/PLICKERT 1976) wurde daraus ein gerasterter Farbauszug für die vier Normalfarben Gelb, Magenta, Cyan und Schwarz hergestellt. Nachher wurde in die Schwarzplatte noch die im Lichtsatz gesetzte Schrift (Titel, Legende) einmontiert. Die Karte ist im Offsetdruckverfahren mit den vier Normalfarben gedruckt.

Dieses Vorgehen erlaubt es, rasch und relativ günstig von einem mehrfarbigen Original eine beliebige Anzahl qualitativ recht guter Drucke herzustellen. Das Verfahren dürfte u. a. für Amtsstellen von Interesse sein, die von handgemalten Originalen für Vernehmlassungsverfahren oder ähnliche Zwecke kurzfristig eine gewisse Anzahl von farbigen Kopien benötigen.

\section{Die Arbeitsplatzstruktur der Stadt Bern 1975}

\subsection{Die Entwicklung der Beschäftigtenzahl 1965-1975}

Im Gegensatz zur Wohnbevölkerung hat sich die Zahl der Beschäftigten mit Arbeitsort in der Stadt Bern bis zum Beginn der jüngsten Rezession noch ständig erhöht. Zählte man anläßlich der Eidgenössischen Betriebszählung 1965 in Bern 112216 Beschäftigte3), so ergab die Eidgenössische Volkszählung 1970 bereits 118051 Beschäftigte4). Die Eidgenössische Betriebszählung 1975 weist 122133 Beschäftigte5) aus, wobei diese Zahl wegen der Rezession um einiges unter dem Höhepunkt der Entwicklung etwa im Jahre 1973 liegen dürfte.

Ein Vergleich der kleinräumigen Entwicklung der Beschäftigten innerhalb der Stadt Bern für die Periode 1965-1975 ist leider nicht möglich, da die Betriebszählung 1975 erstmals für alle Branchen als reine Arbeitsstättenzählung durchgeführt wurde. Als Arbeitsstätte wurde dabei eine örtliche Einheit definiert, in der Güter erzeugt oder repariert oder Dienste aller Art geleistet werden und in der mindestens eine Person erwerbstätig ist. 1965 dagegen konnten für einzelne Branchen (öffentliche Verwaltung z. B.) die Arbeitsstätten eines Betriebes innerhalb der gleichen $\mathrm{Ge}$ meinde noch zusammengefaßt werden.

\subsection{Arbeitsplatzstruktur (ausgewählte Branchen und Gebiete)}

Bezüglich der Feingliederung der Stadt Bern für die Eidgenössische Betriebszählung 1975 sei auf Abschnitt 2 und Tabelle 1 verwiesen. Die in den Abbildungen 7 bis 11 dargestellten Werte stammen aus einer eigenen, quartierweisen Vorauswertung des Statistischen Amtes der Stadt Bern, die sämtliche Beschäftigten umfaßt im Gegensatz zu den bisher publizierten Zahlen des Eidgenössischen Statistischen Amtes, die sich auf Beschäftigte mit 30 und mehr Wochenstunden beschränken.

Für die Stadt Bern als Ganzes ergaben sich folgende Beschäftigtenzahlen6): primärer Sektor 781 oder $0,6 \%$ (1965 0,7\%7)), sekundärer Sektor 38870 oder $31,8 \%$ (1965 41,8\%) und tertiärer Sektor 82482 oder $67,5 \%$ (1965 57,5\%).

Über 25500 oder rund $21 \%$ der Beschäftigten in der Stadt Bern 1975 hatten ihren Arbeitsplatz im engsten Stadtzentrum (Bezirke 4 und 5, schraffierte Fläche in Abb. 7-9), also auf einer Fläche von 42,9 ha (rund $1,2 \%$ der Gesamtfläche ohne Wald der Stadt Bern). Erweitern wir das City-Gebiet um den dritten Bezirk und Teile der Länggasse (Universität, Stadtbach-Ost), des Monbijou (City-West, Gebiet bis Kapellenstraße) und die Bundesgebäude an der Taubenhalde, so kommen wir bereits auf 38800 oder $31,8 \%$ der Beschäftigten in der Stadt Bern. 
Interessant sind auch noch einige Flächenangaben: Von den in der Stadt Bern 1975 erfaßten rund 5 Millionen $\mathrm{m}^{2}$ Bruttogeschoßfläche der Arbeitsstätten (ohne primärer Sektor, PTT, SBB) entfallen 68,9\% auf den tertiären und $31,1 \%$ auf den sekundären Sektor. $13,8 \%$ der genutzten Geschoßfläche der Stadt Bern liegt in den Statistischen Bezirken 4 und 5. Die größten Flächen belegen die Branchen öffentliche Verwaltung (ohne PTT-, SBB-Verwaltung, 11,7\%), Unterricht/ Kirche (10,7\%), Gesundheit/Wohlfahrt (10,5\%), Detailhandel (10,3\%) und Maschinen/Apparate/Fahrzeuge $(8,7 \%)$.

Die Abbildungen 7 bis 10 zeigen die Verteilung der Beschäftigten für vier ausgewählte Branchen. Wie aus Abb. 7 hervorgeht, sind die Arbeitsplätze der Branche Banken/Versicherungen/Krankenkassen, also einer typischen Dienstleistungsbranche, fast ganz im Zentrum der Stadt und den westlich daran anschließenden Cityrandgebieten konzentriert. Erst in jüngster Zeit wurden einzelne Verwaltungsabteilungen in Außenquartiere (vornehmlich im Stadtteil Kirchenfeld-Schoßhalde) verlegt. Die Branche umfaßt mit rund 5000 Beschäftigten $6,7 \%$ der im tertiären Sektor und $4,5 \%$ aller Beschäftigten in der Stadt Bern. Die durchschnittliche Zahl der Beschäftigten pro Arbeitsstätte beträgt 37,0 .

In Abb. 8 ist die Branche Papier/Graphisches Gewerbe dargestellt. Mit rund 4600 Beschäftigten zählt sie zu den mittelgroßen Branchen des sekundären Sektors in Bern (11,9\% der im 2. Sektor Beschäftigten). Die Schwerpunkte der Arbeitsplätze liegen in dieser Branche ringförmig um das Zentrum herum. Zehn Betriebe zählen mehr als 100 Beschäftigte bei einer durchschnittlichen Beschäftigtenzahl von 26,8 Personen pro Arbeitsstätte.

Abb. 9 zeigt die - neben dem Baugewerbe - größte Branche des sekundären Sektors (Maschinen/Apparate/Fahrzeuge, inkl. Reparatur). Mit 10400 Beschäftigten umfaßt sie $26,8 \%$ der im sekundären Sektor und $8,5 \%$ aller Beschäftigten in der Stadt Bern. Die Betriebe der Maschinenindustrie fehlen beinahe gänzlich im Stadtzentrum. Ihre Standorte sind z. T. aus der historischen Entwicklung zu verstehen (Wasserkraft: Matte, Sulgenbach; erste Eisenbahnlinien: Breitenrain, Muesmatt), z. T. handelt es sich um Neuansiedlungen oder Betriebsverlegungen in den großen Industriezonen der Außenquartiere (Bümpliz, Breitfeld). Bei einer durchschnittlichen Beschäftigtenzahl pro Arbeitsstätte von 32,5 weist die Branche vierzehn Arbeitsstätten von mehr als 100 Beschäftigten auf. Das Maximum wird von einem Betrieb der Fernmeldegeräteherstellung in Bümpliz mit über 1800 Personen erreicht.

Als weitere Branche muß in der «Beamtenstadt» Bern natürlich noch die Branche öffentliche Verwaltung (inkl. PTT- und SBB-Verwaltung) dargestellt werden (Abb. 10). Rund 14000 Beschäftigte zählt dabei die Verwaltung von Bund, Kanton und Gemeinde (allgemeine öffentliche Verwaltung, Rechtspflege, Polizei und Feuerwehr, Landesverteidigung, gesetzliche Sozialversicherung), 1400 Beschäftigte entfallen auf die SBB-Verwaltung, 2100 Beschäftigte auf die PTT-General- und Kreisdirektionen. Mit total 17600 Beschäftigten umfaßt die Branche öffentliche Verwaltung 21,3 Prozent der Beschäftigten des tertiären Sektors oder $14,4 \%$ aller Beschäftigten. Die Arbeitsplätze zeigen eine starke Konzentration in der Innenstadt: Allein 4500 Arbeitsplätze liegen im 1. Stadtteil (Bund, Kanton, Stadt, PTT, SBB), weitere in der Länggasse (SBB, Bund), im Stadtteil Mattenhof-Monbijou (Bund, Stadt, SBB, PTT), im Kirchenfeld (Bund), im Stadtteil Breitenrain-Lorraine (Bund, Kanton, PTT). Praktisch keine Arbeitsplätze in der öffentlichen Verwaltung findet man im ganzen Westen und im Südosten der Stadt.

\subsection{Verhältnis Arbeitsplätze/Wohnbevölkerung}

Das Verhältnis Arbeitsplätze (= Beschäftigte am Arbeitsort)/Wohnbevölkerung weist in der Stadt Bern erwartungsgemäß starke Unterschiede auf. Auf der Basis der Betriebszählungsquartiere (siehe Tab. 1 und Fig. 11) gehen die Werte von unter 1 (d.h. weniger als ein Arbeitsplatz auf 100 Wohnplätze im Quartier) bis rund 9000 (90 Arbeitsplätze auf eine Person der Wohnbevölkerung). Der gesamtstädtische Durchschnitt lag 1975 bei 122133 Arbeitsplätzen und einer Wohnbevölkerung von 151277 Personen (Ende September 1975) bei einem Wert von 81. Die mittlere Erwerbsquote betrug 1970 in der Stadt Bern 51,6\% (voll und in Teilzeit Erwerbstätige), d. h. bei einem Wert von rund 50 Arbeitsplätzen pro 100 Wohnplätze wäre in einem Quartier theoretisch ein ausgeglichenes Verhältnis vorhanden, das eine Pendelwanderung erübrigen würde.

Die niedrigsten Werte finden wir in praktisch reinen Wohnquartieren wie Tiefenau, Steigerhubel, Weißenstein, Bümpliz-Kleefeld u. a. Verhältnisse von weniger als 25 Arbeitsplätzen auf 100 Wohnplätze treffen wir in größeren Teilen von Bümpliz und Bethlehem, im südlichen Abschnitt des Stadtteils Mattenhof-Monbijou, im Gebiet der Schoßhalde, in Teilen des Breitfelds und der nördlichen Lorraine und im Gebiet Äußere Enge-Tiefenau-Felsenau, also alles eher periphere Gebiete.

Werte zwischen 25 und 500 kommen in größeren Flächen in den Stadtteilen 1 bis 5 vor, wobei die niedrigeren Werte bis 75 eher in den äußeren Teilen, die Werte zwischen 75 und 500 in den zentrumsnahen Bereichen und entlang der Hauptausfallstraßen (Thunstraße, Moserstraße, Nordring, Länggaßstraße, Monbijoustraße usw.) zu finden sind.

Über 1:5 steigt das Verhältnis Wohnbevölkerung/Arbeitsplätze im Stadtzentrum und in weiteren typischen «Arbeitsplatzquartieren» wie Industriegebieten (Bümpliz-Bodenweid, Breitfeld, Weyermannshaus u. a.) und Verwaltungs-, Schul-, Militär- oder Spitalschwerpunkten (Kirchenfeld, Vordere Länggasse, Kaserne, Insel u. a.). Die höchsten Quartierwerte werden dort er- 


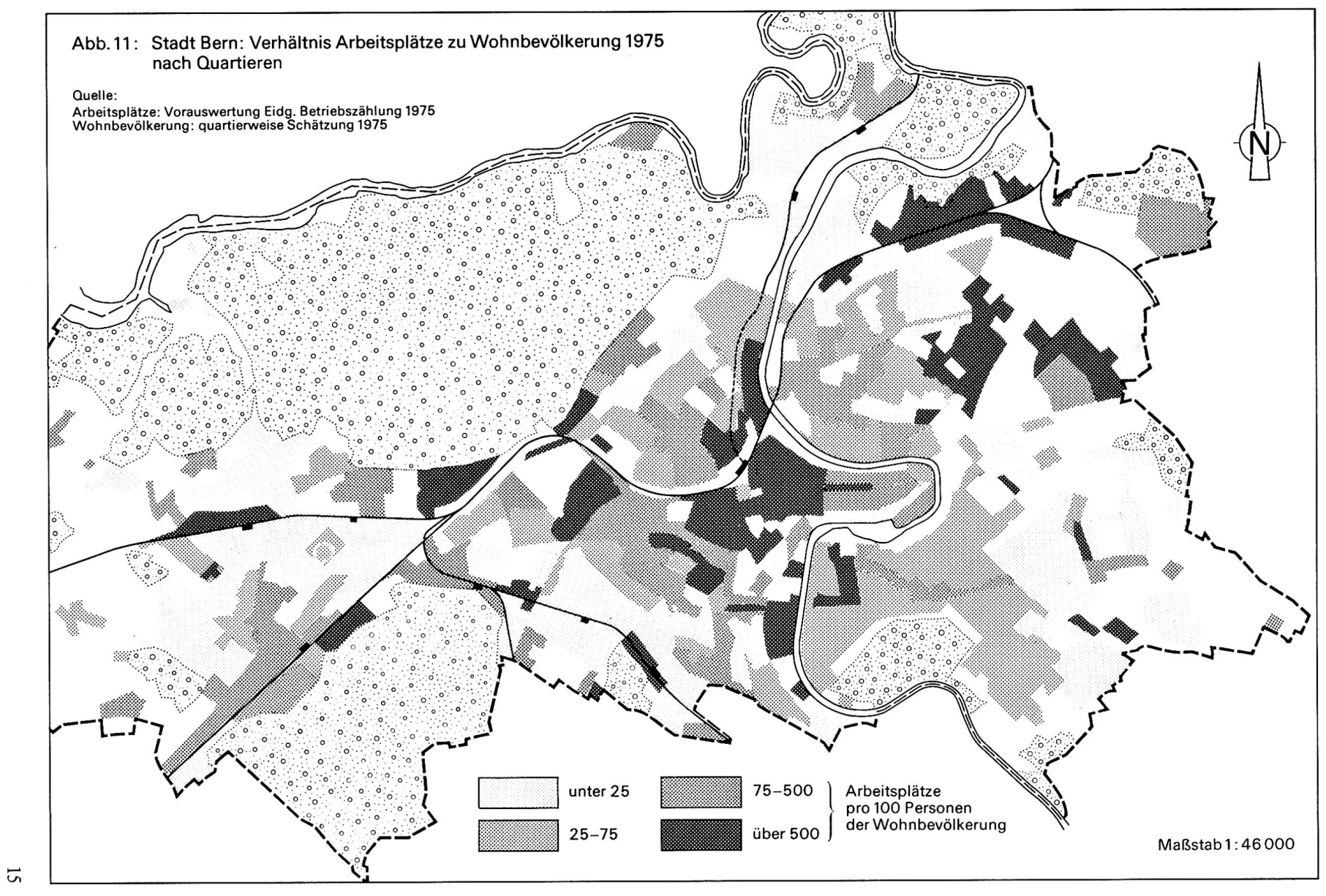


reicht, wo sich die Wohnbevölkerung auf die Hauswarte beschränkt bei einer großen Zahl von Arbeitsplätzen (Marktgasse-Zeughausgasse, City-West, Taubenhalde, Kaserne, Freudenbergerplatz, Wankdorffeldstraße-Nord u. a.).

\section{Zusammenfassung und Schlußbetrachtung}

Der Artikel geht auf die Probleme einer feineren räumlichen Aufgliederung der Stadt Bern ein. Es wird gezeigt, welche Möglichkeiten für eine mikrostatistische Analyse sich aus den Eidg. Volks-, Wohnungsund Betriebszählungen ergeben und in welchen Bereichen das Datenmaterial durch zusätzliche Erhebungen ergänzt werden sollte.

Um ständig über einigermaßen aktuelles Datenmaterial zur kleinräumigen Stadtentwicklung verfügen zu können, sollte über die Großzählungen hinaus eine eigene Einwohner- und Gebäudedatenbank vorhanden sein, die die wichtigsten Angaben über Bestände und Bewegungen periodisch liefern kann.

In Anbetracht der großen Datenmengen kommt einer quantitativen Analyse große Bedeutung zu. Ebenfalls muß der Frage der kartographischen Darstellung der gewonnenen Daten die nötige Beachtung geschenkt werden.

\section{Anmerkungen}

1) Die quantitativen Analysen wurden in Zusammenarbeit mit den Herren Dr. phil. nat. Pierre Hess und lic. rer. pol. Hans Liechti auf der Rechenanlage der Universität Bern durchgeführt. Der Autor möchte auch an dieser Stelle den beiden Herren für ihre Mitarbeit danken.

2) Die Differenz zwischen der Anzahl Quartiere in Tab. 3 und Abb. 6 ergibt sich aus dem Umstand, $\mathrm{da} \beta$ in Abb. 6 nur die in die Cluster-Analyse einbezogenen Quartiere dargestellt sind, während in Tab. 3 auch die nachträglich zugewiesenen Quartiere gezählt werden.

3) Haupt- und nebenberuflich Beschäftigte (aber ohne nebenberuflich Beschäftigte in der öffentlichen Verwaltung, dem öffentlichen Schulwesen, dem Kirchendienst und der Landwirtschaft).

4) Umrechnung der Berufstätigen auf den Arbeitsort nach der Pendlerstatistik; voll und in Teilzeit Erwerbstätige.

5) Voll und in Teilzeit Beschäftigte, gemäß Vorauswertung.

6) In der Vorauswertung 1975 wurde die gleiche Sektoren- und Branchengliederung wie für 1965 angewendet. Die Zahlen sind deshalb nicht in allen Teilen mit den durch das Eidg. Statistische Amt für 1975 publizierten Zahlen vergleichbar.

7) $1965: 100 \%=$ Total der hauptberuflich Beschäftigten.

\section{Literatur}

BETHLEHEM, Broschüre herausgegeben von den Gemeinschaftszentren in Bethlehem, Bern 1975

GÄCHTER, E.: Die demographisch-sozioökonomische Struktur der Stadt Bern 1970 in quartierweiser Gliederung, in: Berner Beiträge zur Stadt- und Regionalforschung, Heft 1/1974, Bern 1974

DERS.: Die demographisch-sozioökonomische Struktur der Region Bern (ohne Stadt Bern) nach Quartieren und Gemeindeteilen 1970, in: Berner Beiträge zur Stadt- und Regionalforschung, Heft 2/1974, Bern 1974 DERS.: Quantitative Untersuchungen zur Quartierstruktur der Stadt Bern 1970, Text- und Beilagenband, Statistisches Amt der Stadt Bern, Bern 1977 (vervielfältigt)

DERS.: Die Gebäude- und Wohnungsstruktur von Stadt und Region Bern 1970 nach Quartieren und Gemeindeteilen

DERS.: Die demographische Struktur der Stadt Bern 1960 in quartierweiser Gliederung (mit Vergleichen zu 1970)

BUCHER, J. und GÄCHTER, E.: Einkommensstruktur und Einkommensverteilung in der Stadt Bern 1970 nach Quartieren. Alle drei Artikel in: Berner Beiträge zur Stadt- und Regionalforschung, Heft 4/1975, Bern 1975 GROSJEAN, G.: Bern, Arealnutzung und bauliche Struktur 1963/67, Karte 1:25000, in: Atlas der Schweiz, Tafel 43, Bern 1968

DERS.: Kanton Bern, Historische Planungsgrundlagen (Planungsatlas Kanton Bern, dritte Lieferung), Bern 1973

HAMM, B.: Die Organisation der städtischen Umwelt, Reihe Soziologie in der Schweiz, Nr. 6, Frauenfeld 1977 HERZOG, H.: Fragen der Einteilung einer Stadt für die Zwecke der Statistik und der Stadtforschung, in: Berner Beiträge zur Stadt-und Regionalforschung, Heft 1/1968, Bern 1968

KILCHENMANN, A. und GÄCHTER, E.: Neuere Anwendungsbeispiele von quantitativen Methoden, Computer und Plotter in der Geographie und Kartographie, in: Geographica Helvetica, 24. Jg., Heft 2, Bern 1969 RAETH,E. und PLICKERT, w.: Kurzfristige Vervielfältigung mehrfarbiger Karten, in: Kartographische Nachrichten, 26. Jg., Heft 3, Bonn 1976

STEINER, D.: Die Faktorenanalyse, ein modernes statistisches Hilfsmittel des Geographen für die objektive Raumgliederung und Typenbildung, in: Geographica Helvetica, 20. Jg., Heft 1, Bern 1965

DERS.: Geographische Raumgliederung und Mustererkennung, in: Beiträge zur heutigen Humangeographie, Geographisches Institut der ETH Zürich, Publ. Nr. 55, Zürich 1975

STATISTISCHES AMT DER STADT BERN: Ergebnisse einer Vorauswertung der Eidgenössischen Betriebszählung 1975 für Stadt und Region Bern, Bern 1976 (vervielfältigt) 